\title{
ZNF492 and GPR149 methylation patterns as prognostic markers for clear cell renal cell carcinoma: Array-based DNA methylation profiling
}

\author{
YONG-JUNE KIM ${ }^{1 *}$, WOOYEONG JANG ${ }^{2 *}$, XUAN-MEI PIAO ${ }^{1}$, HYUNG-YOON YOON ${ }^{1}$, YOUNG JOON BYUN ${ }^{1}$, \\ JI SANG KIM ${ }^{1}$, SUNG MIN KIM ${ }^{1}$, SANG KEUN LEE ${ }^{1}$, SUNG PIL SEO ${ }^{1}$, HO WON KANG ${ }^{1}$, WON TAE KIM ${ }^{1}$, \\ SEOK JOONG YUN ${ }^{1}$, HO SUN SHON ${ }^{3}$, KEUN HO RYU ${ }^{4}$, SANG WON KIM ${ }^{5}$, YUN-SOK HA ${ }^{5}$, \\ GHIL SUK YOON ${ }^{6}$, SANG-CHEOL LEE ${ }^{1}$, TAE GYUN KWON ${ }^{5}$ and WUN-JAE KIM ${ }^{1}$ \\ ${ }^{1}$ Department of Urology, College of Medicine, Chungbuk National University, Cheongju, Chungcheongbuk 28644; \\ ${ }^{2}$ Clinical Genomics Analysis Branch, National Cancer Center, Goyang, Gyeonggi 10408; \\ ${ }^{3}$ Medical Research Institute and ${ }^{4}$ Department of Computer Science, School of Medicine, Chungbuk National University, \\ Cheongju, Chungcheongbuk 28644; Departments of ${ }^{5}$ Urology and ${ }^{6}$ Pathology, School of Medicine, \\ Kyungpook National University, Jung-gu, Daegu 41944, Republic of Korea
}

Received December 13, 2018; Accepted May 2, 2019

DOI: $10.3892 /$ or.2019.7151

\begin{abstract}
The present study aimed to identify novel methylation markers of clear cell renal cell carcinoma (ccRCC) using microarray methylation analysis and evaluate their prognostic relevance in patient samples. To identify cancer-specific methylated biomarkers, microarray profiling of ccRCC samples from our institute $(\mathrm{n}=12)$ and The Cancer Genome Atlas (TCGA) database $(n=160)$ were utilized, and the prognostic relevance
\end{abstract}

Correspondence to: Professor Wun-Jae Kim, Department of Urology, College of Medicine, Chungbuk National University, 1st Chungdae-ro, Seowon-gu, Cheongju, Chungcheongbuk 28644, Republic of Korea

E-mail:wjkim@chungbuk.ac.kr

Professor Tae Gyun Kwon, Department of Urology, School of Medicine, Kyungpook National University, 680 Gukchaebosang-ro, Jung-gu, Daegu 41944, Republic of Korea

E-mail: tgkwon@knu.ac.kr

*Contributed equally

Abbreviations: CBNUH, Chungbuk National University Hospital; ccRCC, clear cell renal cell carcinoma; CI, confidence interval; gDNA, genomic DNA; GPCRs, G protein-coupled receptors; GPR149, G protein-coupled receptor 149; HR, hazard ratio; KIRC, kidney renal clear cell carcinoma; KPNUH, Kyungpook National University Hospital; NC, normal control; PSQ, pyrosequencing; ROC, receiver operating characteristic; AUC, area under the ROC curve; TCGA, The Cancer Genome Atlas; TWIST1, twist family bHLH transcription factor 1; ZNF492, zinc finger protein 492

Key words: carcinoma, methylation, microarray analysis, prognosis, renal cell of candidate genes were investigated in another TCGA dataset $(n=153)$. For validation, pyrosequencing analyses with ccRCC samples from our institute $(n=164)$ and another $(n=117)$ were performed and the potential clinical application of selected biomarkers was examined. We identified $22 \mathrm{CpG}$ island loci that were commonly hypermethylated in ccRCC. Kaplan-Meier analysis of TCGA data indicated that only 4/22 loci were significantly associated with disease progression. In the internal validation set, Kaplan-Meier analysis revealed that hypermethylation of two loci, zinc finger protein 492 (ZNF492) and G protein-coupled receptor 149 (GPR149), was significantly associated with shorter time-to-progression. Multivariate Cox regression models revealed that hypermethylation of ZNF492 [hazard ratio (HR), 5.44; $\mathrm{P}=0.001$ ] and GPR149 (HR, 7.07; $\mathrm{P}<0.001)$ may be independent predictors of tumor progression. Similarly, the methylation status of these two genes was significantly associated with poor outcomes in the independent external validation cohort. Collectively, the present study proposed that the novel methylation markers ZNF492 and GPR149 could be independent prognostic indicators in patients with ccRCC.

\section{Introduction}

Renal cell carcinoma (RCC) is a common malignancy of the genitourinary system, and is classified into various subtypes based on histopathological characteristics and each subtype has a diverse prognosis $(1,2)$. From a clinical viewpoint, three main RCC subtypes are important in research: Clear cell RCC (ccRCC; 80-90\% cases), papillary RCC (10-15\% cases), and chromophobe RCC $(4-5 \%$ cases) $(1,2)$. The development of RCC is complex, and numerous parameters, including anatomical, histological, clinical and molecular factors contribute to disease outcome. Despite great improvements in understanding the major molecular mechanisms involved 
in RCC, none of the conventional or molecular markers have demonstrated satisfactory sensitivity and specificity to be considered as a prognostic indicator of $\operatorname{RCC}(1,2)$. Thus, the majority of studies have focused on the development of suitable biomarkers to improve diagnosis and prognostication in RCC.

As a result of recent advancements in understanding of epigenetic modifications, DNA methylation is now regarded as one of the key findings in the development and progression of cancer. In RCC, several DNA methylation biomarkers associated with tumor development, histological subtypes, prognosis and response to therapy have been reported (3-13); however, the majority of these markers were identified in cohorts examined in single-center studies with variable results. Therefore, their use in routine clinical practice is limited. The development of high-throughput bioinformatics technology, such as genome-wide methylation pattern analysis, now enables tumor-specific novel molecular markers to be identified from numerous samples that can readily be compared with other cohorts via database sharing.

Establishing the association between biomarkers and clinical outcomes in RCC is likely to prove useful for developing effective treatment strategies and improving clinical management. The aim of the present study was to identify novel biomarkers that could predict the outcomes of RCC using genome-wide DNA methylation microarray data derived from patients analyzed in our institute and from The Cancer Genome Atlas (TCGA), and to evaluate their clinical application in ccRCC cases with long-term follow-up.

\section{Materials and methods}

Subjects and sample collection. A total of 355 human kidney specimens were obtained from patients (age range: 30-83 years, sample collection: February 1999 to September 2015) with primary, histologically-confirmed ccRCC who underwent radical or partial nephrectomy from two independent institutes including: i) 201 human kidney specimens [164 ccRCC and 37 matched normal control (NC)] from Chungbuk National University Hospital (CBNUH); and ii) 154 human kidney specimens (117 ccRCC and 37 matched NC) from Kyungpook National University Hospital (KPNUH). To enhance the homogeneity of the study population, patients were enrolled into the study according to the following criteria: i) clinical and pathological stage T1-T4 without lymph node or distant metastasis; and ii) a minimum follow-up period of 3 months. Patients whose samples had a positive surgical margin at final pathology were not included to avoid bias in survival analysis. The pathology of samples was independently reviewed by a pathologist unaware of the intended use of the clinical data. The specimens were obtained from the CBNUH and KPNUH, members of the National Biobank of Korea, which is supported by the Ministry of Health, Welfare and Family Affairs. The collection and analysis of all samples were approved by the Institutional Review Board of the CBNUH (approval no. 2010-01-001) and KPNUH (approval no. KNUMC 2016-05-021). Informed consent was obtained from each patient. The study design and validation strategies were presented in Fig. 1. Tumors were categorized based on pathological stage and histological subtype, as previously described $(14,15)$. Nuclear differentiation was graded according to the Fuhrman Nuclear Grade system (16). All patients were followed-up and managed according to standard recommendations (1). Progression was defined as lymph node involvement and/or metastatic disease as determined by a computed tomography scan or bone scan.

DNA methylation profiling. Genomic DNA (gDNA) from patient's samples was extracted according to the manufacture's protocol by using the Wizard Genomic DNA Purification System (Promega Corporation, Madison, WI, USA). Bisulfite-modified gDNA was prepared using the EZ DNA Methylation-Lightning kit (Zymo Research, Irvine, CA, USA) according to the manufacturer's instructions. The methylation status was assayed using the Infinium HumanMethylation450 BeadChip array (Infinium Methylation 450K; Illumina, Inc., San Diego, CA, USA), which assesses the methylation status of $>480,000 \mathrm{CpG}$ sites distributed across the whole genome. A total of 24 matched DNA samples (pairs of NC and ccRCC from 12 patients) were used for DNA methylation profiling. Fluorescence signals corresponding to $\mathrm{C}$ - or T-nucleotides were measured (GenomeStudio Methylation Module, V1.8, Illumina, Inc., San Diego, CA, USA), and the data were used to assign a quantitative measure of methylation of specific $\mathrm{CpG}$ islands ( $\beta$ value): Ranging from 0 (complete unmethylation) to 1 (complete methylation). The complete sets of microarray methylation data are available online (http://www.ncbi.nlm. nih.gov/geo/; accession no. GSE92482.

Array profiling data from TCGA. For comparisons with the array data from the selected $\mathrm{CBNUH}$ patient samples and to investigate the prognostic relevance of the identified methylated $\mathrm{CpG}$ islands, the TCGA kidney renal clear cell carcinoma (KIRC) level 3 data set, also obtained using the Infinium HumanMethylation450 BeadChip, was investigated (https:// cancergenome.nih.gov/). Methylation profiles of $313 \mathrm{ccRCC}$ patient samples were available within the KIRC dataset. The dataset was divided into two groups: i) Training set, 160 pairs of ccRCC and NC matched samples; and ii) test set, an addition 153 ccRCC samples without matched NC samples. The training set data were used for comparisons with the candidate $\mathrm{CpG}$ sites identified with the CBNUH samples, and the test set data were used for assessing the prognostic relevance of those candidates. Baseline characteristics of the KIRC patients are provided in Table SI.

Pyrosequencing (PSQ) analysis. The DNA methylation status of the candidate $\mathrm{CpG}$ island loci identified using the Infinium HumanMethylation450 array were specifically assessed by PSQ analysis of 331 human kidney samples (CBNUH, 152 ccRCC and 25 matched NCs; KPNUH, 117 ccRCC and 37 matched NCs) using a PyroMark Q96 ID instrument (Qiagen, Inc., Valencia, CA, USA) according to the manufacturer's instructions. Each primer was designed using Pyrosequencing Assay Design Software v2.0 (Qiagen, Inc.). PSQ primers were designed to encompass the $\mathrm{CpG}$ island loci determined from the Illumina Infinium array. The primer sequences and amplification conditions are described in Table SII. 


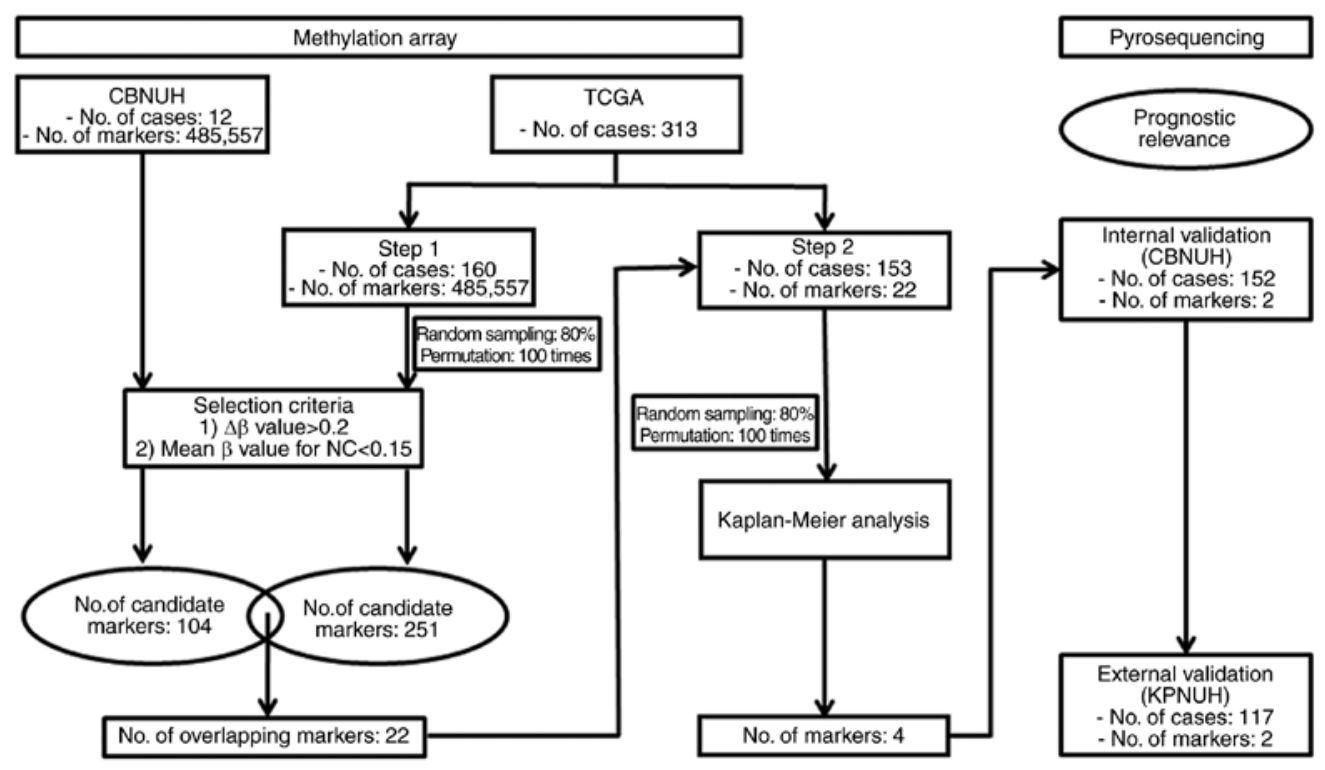

Figure 1. Study design and validation strategies. Genome-wide microarray methylation profiles of ccRCC and corresponding NC samples from CBNUH and TCGA cases were compared to identify ccRCC-specific methylated genes. CBNUH cases: pairs of ccRCC and normal tissue from 12 patients; TCGA cases: pairs of ccRCC and normal control samples from 160 patients. Step 1, TCGA training set; step 2, TCGA test set. Random sampling: 80\%; Permutation: 100 times. Pyrosequencing assays used to validate the candidate markers in CBNUH and KPNUH cases. ccRCC, clear cell renal cell carcinoma; CBNUH, Chungbuk National University Hospital; TCGA, The Cancer Genome Atlas; KPNUH, Kyungpook National University Hospital.

Statistical analysis. The levels of DNA methylation were normalized using quantile normalization in R (v2.10.0, http:// www.r-project.org/). The criteria applied for the selection of ccRCC-specific methylated $\mathrm{CpG}$ island loci were as follows: i) a difference in the levels of DNA methylation between ccRCC and $\mathrm{NC}(\Delta \beta$ value) $>0.2$; and ii) a mean $\beta$-value for $\mathrm{NC}<0.15$. Using the same selection criteria, the training set from the TCGA data was used for comparisons with genes identified from CBNUH ccRCC samples. With the same selection criteria of CBNUH samples, a permutation test (80\% random sampling; 100 times) was conducted to reduce unduly identified $\mathrm{CpG}$ island loci. Only $\mathrm{CpG}$ island loci that possessed $>50$-times difference were selected for further analysis. Among the candidate $\mathrm{CpG}$ island loci identified, those common to the CBNUH ccRCC samples and the TCGA training set were selected as candidate prognostic indicators of ccRCC. The prognostic relevance of candidate $\mathrm{CpG}$ island loci was explored with the TCGA test dataset using Kaplan-Meier analysis (100 times permutation, $80 \%$ sampling) and those loci that had statistical significance $>50$ times were regarded as targets for further validation with ccRCC samples by PSQ.

The differences in continuous variables between groups were assessed using a two-sample t-test or ANOVA trend analyses using polynomial contrasts and data were presented as the mean \pm standard deviation. Receiver operating characteristic (ROC) curves were used to estimate the capability of candidate markers in predicting the of progression of disease, and to determine the optimal cut-off point for dividing patients into subgroups (hypomethylation or hypermethylation) with the highest combined sensitivity and specificity. A multivariate Cox proportional hazards regression model was used to evaluate the prognostic value of candidate markers adjusted for well-known clinicopathological factors (sex, age, body mass index, grade and stage). Statistical analysis was performed using SPSS software v21.0 (IBM
Table I. Baseline characteristics of the patients enrolled in the present study.

\begin{tabular}{lcc}
\hline Variables & $\begin{array}{c}\text { Microarray } \\
(\mathrm{n}=12)\end{array}$ & $\begin{array}{c}\text { Pyrosequencing } \\
(\mathrm{n}=152)\end{array}$ \\
\hline Age (years, mean $\pm \mathrm{SD})$ & $57.3 \pm 11.3$ & $64.3 \pm 13.8$ \\
Gender [n (\%)] & & \\
Male & $8(66.7)$ & $110(72.4)$ \\
Female & $4(33.3)$ & $42(27.6)$ \\
BMI kg/m ${ }^{2}$ (mean $\left.\pm \mathrm{SD}\right)$ & $25.9 \pm 3.3$ & $24.6 \pm 3.6$ \\
Fuhrman grade, [n (\%)] & & \\
G1 & $2(16.7)$ & $34(22.4)$ \\
G2 & $7(58.3)$ & $68(44.7)$ \\
G3 & $2(16.7)$ & $45(29.6)$ \\
G4 & $1(8.3)$ & $5(3.3)$ \\
T stage, [n (\%)] & & \\
T1 & $4(33.3)$ & $112(73.7)$ \\
T2 & $4(33.3)$ & $14(9.2)$ \\
T3 & $4(33.3)$ & $26(15.1)$ \\
T4 & - & $3(2.0)$ \\
Progression-free survival & & $27.2(3.0-188.9)$ \\
(months) Median (range) & & \\
Progression [n (\%)] & & $109(71.8)$ \\
No & & $43(28.2)$ \\
Yes & & \\
\hline
\end{tabular}

BMI, body mass index; SD, standard deviation.

Corp. Armonk, NY, USA) and R v2.12.1 software. $\mathrm{P}<0.05$ was considered to indicate a statistically significant difference. 

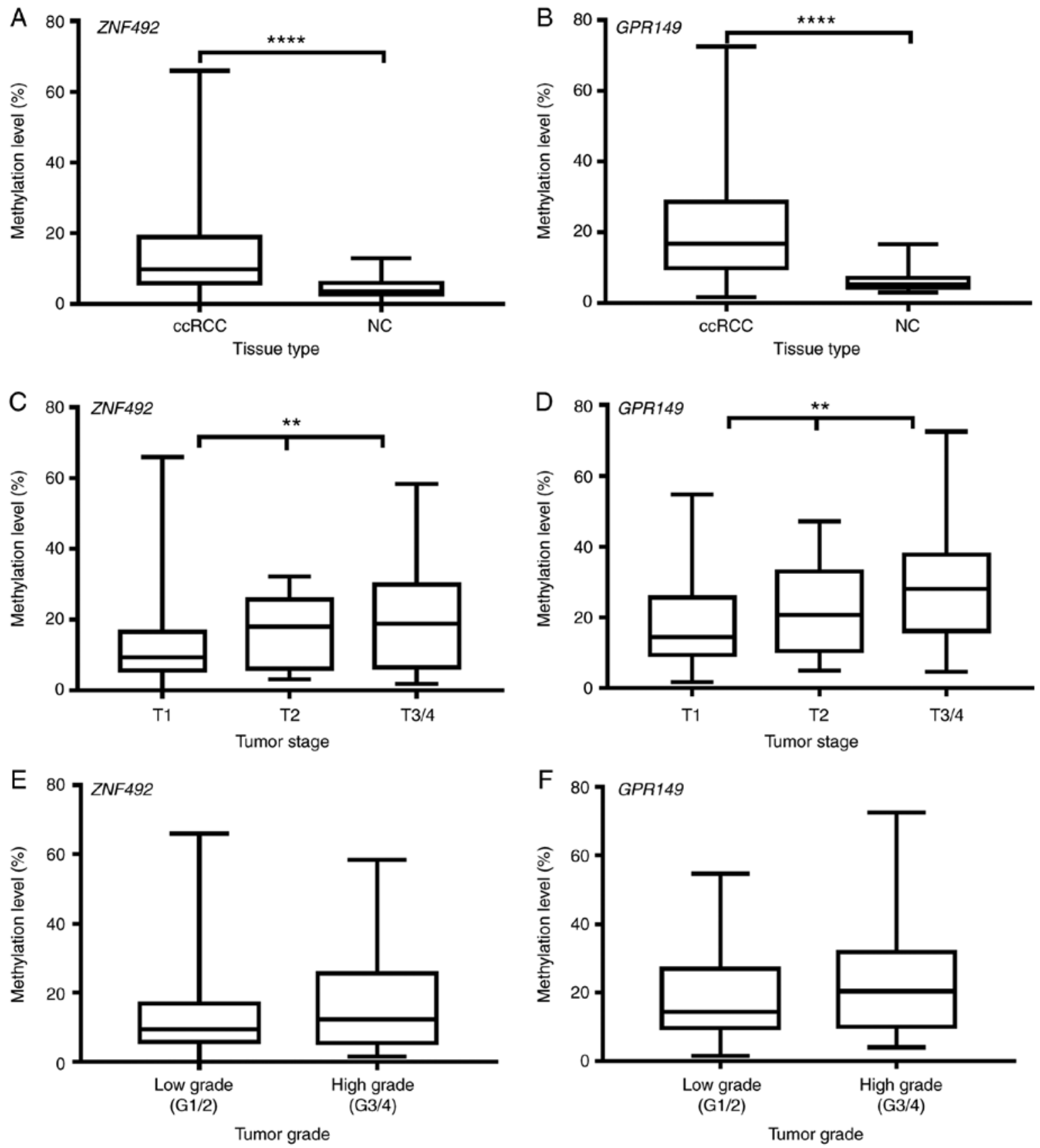

Figure 2. Association between methylation markers and clinicopathological characteristics. (A and B) The methylation levels of ZNF492 and GPR149 were significantly increased in the ccRCC specimens than in the NCs ( $\left.{ }^{* * * *} \mathrm{P}<0.05\right)$. (C and D) Increased methylation levels of ZNF492 and GPR149 were significantly associated with advanced pathological T stage ( $(* * \mathrm{P}<0.05)$. Methylation levels of (E) ZNF492 and (F) GPR149 exhibited a tendency to increase with higher grade, but the differences in methylation were not statistically significant ( $\mathrm{P}=0.059,0.052$, respectively). ccRCC, clear cell renal cell carcinoma; GPR149, G protein-coupled receptor 149; NC, normal control; ZNF492, zinc finger protein 492.

\section{Results}

Baseline characteristics. The baseline characteristics of the 164 CBNUH patients with ccRCC were presented in Table I. The mean age and median progression-free survival period of the patients for PSQ were $64.3 \pm 13.8$ years and 27.2 months (ranging 3.0-188.9 months), respectively; 43 patients (28.2\%) experienced disease progression.

Identification of differentially methylated genes in ccRCC and $N C$. Genome-wide microarray methylation profiles of ccRCC samples and corresponding NC samples from $12 \mathrm{CBNUH}$ cases were compared to identify ccRCC-specific methylated genes. After applying selection criteria $(\Delta \beta$ value $>0.2$ and mean $\beta$-value in $\mathrm{NC}<0.15), 104$ unique $\mathrm{CpG}$ island loci that were hypermethylated in ccRCC compared with the NCs were identified (Table SIII). The 104 selected candidate markers were then validated by comparing with the TCGA training data set from 160 pairs of ccRCC and matched NC samples subjected to the aforementioned selection criteria. In the TCGA training set, $251 \mathrm{CpG}$ island loci were hypermethylated in ccRCC compared with the NCs (Table SIV). Of these, $22 \mathrm{CpG}$ island loci overlapped with those identified as ccRCC-specific in the 12 CBNUH cases (Table SV). The prognostic importance of 
A

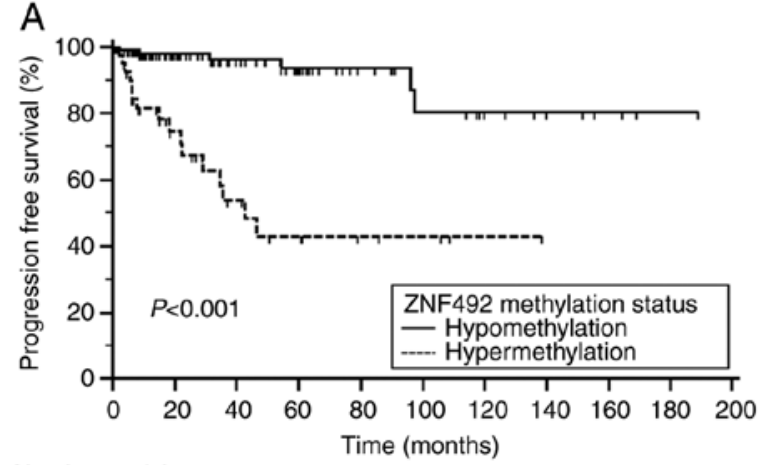

Number at risk

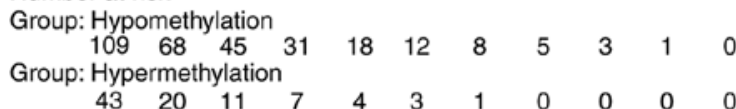

$\mathrm{B}$

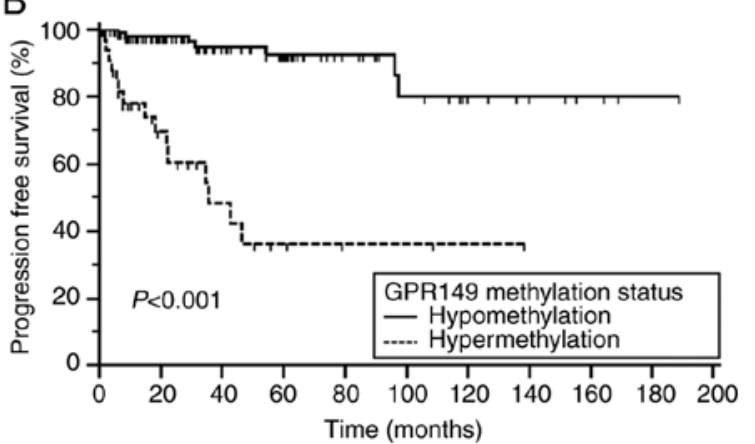

Number at risk

Group: Hypomethylation

$\begin{array}{ccccccccccc}116 & 73 & 48 & 34 & 20 & 13 & 8 & 5 & 3 & 1 & 0\end{array}$

$\begin{array}{lllllllllll}36 & 15 & 8 & 4 & 2 & 2 & 1 & 0 & 0 & 0 & 0\end{array}$

Figure 3. Kaplan-Meier analysis of the Chungbuk National University Hospital cohort. Kaplan-Meier estimates of the probability of progression according to methylation status (identified by pyrosequencing) in 152 patients with clear cell renal cell carcinoma. (A) ZNF492. (B) GPR149. GPR149, G protein-coupled receptor 149; ZNF492, zinc finger protein 492.

the 22 candidate $\mathrm{CpG}$ island loci were then examined with the TCGA test dataset. In the 100-times permutation test of the Kaplan-Meier analysis, 4/22 CpG island loci were selected as targets for validation by PSQ using $152 \mathrm{CBNUH}$ ccRCC samples. The candidate genes at the four loci were as follows: Zinc finger protein 492 (ZNF492; at two CpG island loci), twist family bHLH transcription factor 1 (TWIST1), and G protein-coupled receptor 149 (GPR149).

PSQ analysis and internal validation of prognostic relevance in the clinical samples. To verify the prognostic relevance of candidate genes using a different methodology in a larger, different set of samples, we performed PSQ analyses on bisulfite-modified gDNA obtained from $177 \mathrm{CBNUH}$ human kidney specimens (152 ccRCC and 25 matched NC). PSQ analysis was performed on two of the four candidate $\mathrm{CpG}$ island regions in the present study, including one of the two ZNF492 loci (Infinium HumanMethylation450 target ID, $\operatorname{cg} 01485075)$ and GPR149 (cg00046499). Analysis of the candidate gene TWIST1 (cg26818735) was not possible due to technical variability across multiple PSQ assays of complex sequences of the homopolymer for PSQ.

To examine the association between methylation patterns and clinicopathological factors, methylation values for each gene were compared between samples. The methylation levels of ZNF492 and GPR149 were significantly higher in the ccRCC specimens than in the NCs $(\mathrm{P}<0.05)$. Increased methylation levels of ZNF492 and GPR149 were significantly associated with advanced pathological $\mathrm{T}$ stage $(\mathrm{P}<0.05)$. Decreased methylation levels of ZNF492 were associated with higher body mass index; however, no significant association was observed with GPR149. The methylation levels of ZNF492 and GPR 149 exhibited significantly increased methylation compared with control samples and had a tendency to increase with higher grade; however, differences between low and high grade tumors in GPR 149 methylation were not statistically significant (Fig. 2, Table SVI).

The value of candidate methylation markers for the prediction of disease progression was determined using the area under the ROC curve (AUC). The AUC values for
ZNF492 and GPR149 were 0.716 [95\% confidence interval (CI), 0.636-0.787; $\mathrm{P}<0.001]$ and 0.781 (95\% CI, 0.707-0.842; $\mathrm{P}<0.001$ ), respectively (Fig. S1). To validate the prognostic relevance of candidate methylation markers, the methylation values of ZNF492 and GPR149 were dichotomized (hypomethylation or hypermethylation) using optimal cut-off points (ZNF492: 18.11 and GPR149: 29.31), and survival analysis was performed. Kaplan-Meier estimates identified significant differences in time-to-progression according to the methylation status of ZNF492 and GPR149 (log-rank test, $\mathrm{P}<0.05$; Fig. 3). In the multivariate Cox regression analyses, the methylation status of ZNF492 [hazard ratio (HR), 5.44; 95\% CI, 2.04-14.53; $\mathrm{P}=0.001]$ and of GPR149 (HR, 7.07; 95\% CI, $2.65-18.89 ; \mathrm{P}<0.001)$ was suggested as independent predictors of disease progression (Table II).

External validation of prognostic relevance in the clinical samples. The validity of two genes as methylation markers in ccRCC was also evaluated using an independent set of PQS data from KPNUH samples, which included ccRCC $(n=117)$ and matched NC $(n=37)$ tissues (Table SVII). The association between the two methylation markers and clinicopathological characteristics revealed similar results to those of the CBNUH dataset (Table SVIII). The AUC values for ZNF492 and GPR149 were 0.717 [95\% CI, 0.542-0.891; $\mathrm{P}=0.014$ ] and 0.866 (95\% CI, 0.746-0.986; P<0.001), respectively (Fig. S1). Patients with hypermethylated genes had shorter durations of progression than those of hypomethylated status (log-rank test, $\mathrm{P}<0.05$, respectively; Fig. 4). According to the Cox multivariate regression analysis, the methylation status of ZNF492 (HR), 3.89; 95\% CI, 1.04-14.61; $\mathrm{P}=0.044]$ and of GPR149 (HR, 33.67; 95\% CI, 5.87-192.88; $\mathrm{P}<0.001)$ were significantly associated with disease progression (Table SIX).

\section{Discussion}

DNA methylation is a crucial mechanism for the development and progression of cancer. The methylation status of various genes can be used as a biomarker for risk assessment, early detection, prediction of prognosis, and therapy response in 
Table II. Univariate and Multivariate Cox regression analyses for determining disease outcome based on the methylation status of each gene.

\begin{tabular}{|c|c|c|c|c|c|c|c|c|}
\hline \multirow[b]{3}{*}{ Variables $^{\mathrm{a}}$} & \multicolumn{4}{|c|}{ Model $1^{\mathrm{b}}$} & \multicolumn{4}{|c|}{ Model $2^{c}$} \\
\hline & \multicolumn{2}{|l|}{ Univariate } & \multicolumn{2}{|c|}{ Multivariate } & \multicolumn{2}{|l|}{ Univariate } & \multicolumn{2}{|c|}{ Multivariate } \\
\hline & $\operatorname{HR}(95 \% \mathrm{CI})^{\mathrm{a}}$ & P-value & $\mathrm{HR}(95 \% \mathrm{CI})^{\mathrm{b}}$ & P-value & $\operatorname{HR}(95 \% \mathrm{CI})^{\mathrm{a}}$ & P-value & $\mathrm{HR}(95 \% \mathrm{CI})^{\mathrm{b}}$ & P-value \\
\hline Age & $1.02(0.98-1.05)$ & 0.373 & - & - & $1.02(0.98-1.05)$ & 0.373 & - & - \\
\hline Sex & $0.779(0.30-2.00)$ & 0.603 & - & - & $0.779(0.30-2.00)$ & 0.603 & & \\
\hline BMI & $0.37(0.16-0.86)$ & 0.021 & $0.35(0.12-1.04)$ & 0.058 & $0.37(0.16-0.86)$ & 0.021 & $0.22(0.07-0.65)$ & 0.007 \\
\hline Grade & $5.39(2.19-13.23)$ & $<0.001$ & $5.39(2.19-13.23)$ & $<0.001$ & $5.39(2.19-13.23)$ & $<0.001$ & $1.85(0.55-6.29)$ & 0.323 \\
\hline Stage & & $<0.001$ & & 0.001 & & $<0.001$ & & 0.003 \\
\hline $\mathrm{T} 1$ & Reference & - & Reference & & Reference & - & Reference & \\
\hline $\mathrm{T} 2$ & $5.65(1.71-18.67)$ & - & $6.37(1.56-25.71)$ & & $5.65(1.71-18.67)$ & - & $7.43(1.89-29.28)$ & \\
\hline $\mathrm{T} 3 / 4$ & $10.33(3.81-28.01)$ & - & $7.75(2.56-23.47)$ & & $10.33(3.81-28.01)$ & - & $5.78(1.77-18.94)$ & \\
\hline ZNF492 & $9.32(3.62-23.91)$ & $<0.001$ & $5.44(2.04-14.53)$ & 0.001 & - & - & - & - \\
\hline GPR149 & - & - & - & - & $10.51(4.26-25.93)$ & $<0.001$ & $7.07(2.65-18.89)$ & $<0.001$ \\
\hline
\end{tabular}

aVariables were categorized as follows: Median age (low vs. high), sex (male vs. female), BMI ( $<22.9$ vs. $\left.\geq 23 \mathrm{~kg} / \mathrm{m}^{2}\right)$, tumor grade $(\mathrm{G} 1 / 2$ vs. G3/4), and methylation status (hypomethylation vs. hypermethylation). ${ }^{\mathrm{b}}$ Multivariate analysis included clinicopathological variables and the methylation status of ZNF492. 'Multivariate analysis included clinicopathological variables and the methylation status of GPR149. BMI, body mass index; CI, confidence interval; GPR149, G protein-coupled receptor 149; HR, hazard ratio; ZNF492, zinc finger protein 492.
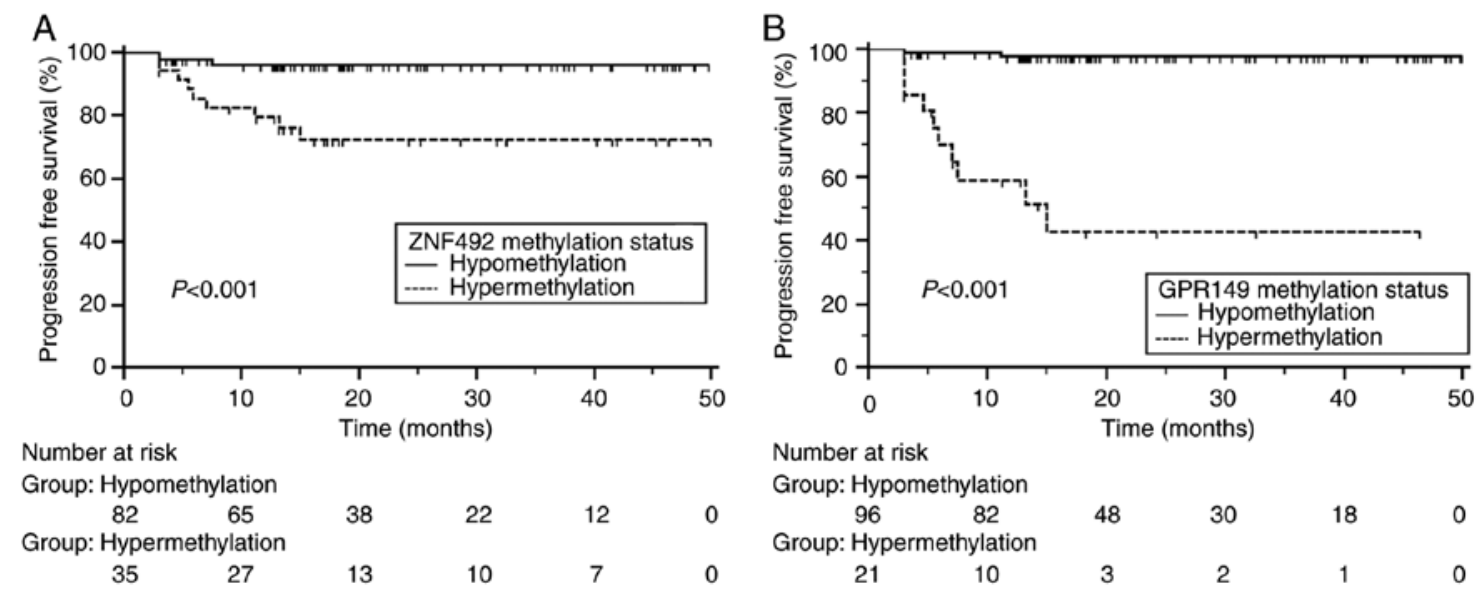

Figure 4. Kaplan-Meier analysis of the Kyungpook National University Hospital cohort. Kaplan-Meier estimates of the probability of progression according to methylation status (identified by pyrosequencing) in 117 patients with clear cell renal cell carcinoma. (A) ZNF492. (B) GPR149. GPR149, G protein-coupled receptor 149; ZNF492, zinc finger protein 492.

numerous types of cancer $(17,18)$. In the present study, novel methylation markers that were able to predict the likelihood of disease progression were identified based on genome-wide DNA methylation array datasets of clinical samples from our own institute and from TCGA. Close associations with unfavorable characteristics of disease, including advanced stage were noted. Furthermore, the methylation status of each of these markers was revealed to be an independent indicator of progression in two cohorts of patients with ccRCC.

Increasing evidence has indicated that the methylation status of individual or combinations of genes are associated with different outcomes in patients with RCC and may serve as independent indicators of prognosis (3-6). In a study of 179 patients who underwent radical or partial nephrectomy for ccRCC, Ras association domain-containing protein 1 methylation status was significantly associated with higher grade and advanced stage. Additionally, this gene served as an independent variable for the prediction of cancer-specific survival in patients with ccRCC (6). Similarly, another study revealed that the combined methylation status of collagen $\alpha-1$ (XIV) and basonuclin was independently associated with a poorer prognosis, and served as a better prognostic indicator than tumor stage or grade (4).

Previously, microarray-based clinical studies have sought novel molecules that could enhance diagnostic and prognostic ability alone or with conventional biomarkers (7-11). In a genome-wide study with kidney tissues from 96 patients, a set of DNA methylation markers was demonstrated to reliably 
discriminate between malignant and benign lesions, and classical histological subtypes (7). In addition, these findings were validated using a microarray data set from TCGA comprising $>1,000$ kidney samples. In addition, other studies have suggested the possible roles of methylation signatures for the detection and prognostic prediction of RCC (8-11). Microarray-based DNA methylation profiling may therefore provide insight into the disease and, most importantly, reveal biomarkers for the detection and prognostication of RCC. In the present study, using methylation profiles of our clinical samples and those in TCGA, we selected $\mathrm{CpG}$ island loci commonly hypermethylated in ccRCC compared with NCs. The potential of these candidate loci as indicators of prognosis was first explored in a test set of TCGA data and independently validated with a different technique (PSQ) in two independent ccRCC cohorts with long-term follow-up. Our methodological approach for gene selection and prognostic validation using multiple steps with different independent data sets was selected to enhance the reliability of our results as much as possible. Furthermore, limiting patient enrollment to those with ccRCC increased the homogeneity of the samples, which may enhance the likelihood of identifying differences. Our candidate markers are likely to be applicable across various ethnicities as they were determined from data derived from Western and Eastern populations. The results of the present study are therefore promising; however, further investigation is required to determine the false prediction rate of these markers in particular.

Limitations of the present study must be addressed. Only 12 samples were used for genome-wide microarray methylation profiling to identify candidate markers. To overcome the limited sample number, a large TCGA dataset was utilized simultaneously. Additionally, validation was performed using PSQ in a larger set of different samples from our institute and others. These types of multiple validation strategies with different datasets may improve the reliability of our results. Additionally, the effects of methylation status on mRNA and protein expression should have been evaluated. Gene expression and immunohistochemical analysis could not be performed due to the lack of available tissue samples. Although the association between alterations in methylation and gene expression of selected markers was not determined in the present study, preliminary data analysis with TCGA data revealed that the expression of ZNF492 was significantly lower in cancer tissues than in NC samples (Fig. S2). Furthermore, our study investigated hypermethylation genes instead those hypomethylated. Global hypomethylation or locus-specific hypermethylation in $\mathrm{CpG}$ island-rich promoters is a common phenomenon of cancer. Numerous sites of hypomethylation that vary between normal and RCC tissues could be identified with a microarray dataset. In the present study, we aimed to analyze 2-5 genes at most with validation in clinical samples. Providing that too many candidate genes are selected, it would be difficult to validate their clinical relevance in clinical samples. In this regard, we focused on the hypermethylation rather than the hypomethylation of genes.

To the best of our knowledge, this is the first report to demonstrate that ZNF492 and GPR149 act as methylation-induced prognostic indicators in RCC. Currently, limited information regarding the specific function of these genes is available in the literature. ZNF492, also known as $Z N F 115$, is a member of the zinc finger protein family and is located at $19 \mathrm{p} 12$. There are eight different classes of zinc finger motifs and each class has a distinct biological role (19). Evidence accumulated over several decades has demonstrated crucial roles for zinc finger proteins in the initiation and progression of cancer in numerous type of cancers (20-24). As an example of the role of methylation, one zinc finger family member ZNF545 was reported to serve as a tumor suppressor by inducing cell apoptosis, inhibiting ribosome biogenesis, and suppressing nuclear factor- $\varkappa \mathrm{B}$ and activator protein-1 signaling in nasopharyngeal, esophageal, lung, gastric, colon, and breast cancer upon methylation (21). In addition, a combination of methylated $\mathrm{CpG}$ island sites corresponding to those of zinc finger genes was able to predict survival outcome in patients with gastric cancer (22). GPR149 is a member of the G protein-coupled receptor (GPCR) family, which is located at 3q25.2. GPCRs comprise a large family of cell-surface receptors and control various features of tumorigenesis, as well as many cancer-associated signaling pathways $(25,26)$. With aid of genome-wide approaches, numerous biomolecules have recently emerged as novel biomarkers in several human tumors; however, in the majority of cases, the association between a specific disease and a putative biomarker has not been yet determined. Similarly, we have not investigated the functional roles of ZNF492 and GPR149 in the tumorigenesis and progression of RCC, which poses as a limitation of the present study. However, establishing novel biomarkers, even of unknown function, and the potential application in the detection, prognostic prediction and use as therapeutic targets is likely to prove valuable in various types of human disease. Therefore, functional studies are required to determine the full potential of the putative biomarkers identified in the present study.

\section{Acknowledgements}

The abstract was presented at the 66th Annual Meeting of the Korean Urological Association Oct 12-Oct 142016 in Seoul, published as abstract no. O-047 in Korean Urological Association Abstracts: 2016.

\section{Funding}

This study was supported by the Basic Science Research Program through the National Research Foundation of Korea funded by the Ministry of Education (grant nos. 2015R1D1A1A01057786 and 2018R1D1A1B07043906).

\section{Availability of data and materials}

The datasets generated and analyzed during the current study are available in the GEO repository, https://www.ncbi.nlm.nih.gov/ geo/query/acc.cgi?token=azovkgyunvcdrij\&acc=GSE92482.

\section{Authors' contributions}

YJK, SCL, TGK and WJK made substantial contributions to the concept of the present study. YJK, WJ, TGK and WJK conducted the experiments. Validation of the data was 
conducted by YJB, JSK and SPS. YJK, WJ, HYY, SMK, SKL, SPS, HWK, WTK, SJY, HSS, KHR, SWK, YSH and GSY performed data analysis. YJK, WJ, YJB and JSK collected data. YJK drafted the manuscript. YJK, WJ, XMP, HYH, SMK, SKL, HWK, WTK, SJY, HSS, KHR, SWK, YSH, GSY, SCL, TGK and WJK reviewed the manuscript. SCL, TGK and WJK provided guidance and supervision. YJK acquired funding for the study.

\section{Ethics approval and consent to participate}

The collection and analysis of all samples were approved by the CBNUH (approval no. 2010-01-001) and KPNUH (approval no. KNUMC 2016-05-021). Institutional Review Board and informed consent was obtained from each subject. The study methodologies conformed with the standards set by the Declaration of Helsinki.

\section{Patient consent for publication}

Not applicable.

\section{Competing interests}

The authors declare they have no competing interests.

\section{References}

1. Ljungberg B, Bensalah K, Canfield S, Dabestani S, Hofmann F, Hora M, Kuczyk MA, Lam T, Marconi L, Merseburger AS, et al: EAU guidelines on renal cell carcinoma: 2014 update. Eur Urol 67: 913-924, 2015.

2. Srinivasan R, Ricketts CJ, Sourbier C and Linehan WM: New strategies in renal cell carcinoma: Targeting the genetic and metabolic basis of disease. Clin Cancer Res 21: 10-17, 2015.

3. Eggers H, Steffens S, Grosshennig A, Becker JU, Hennenlotter J, Stenzl A, Merseburger AS, Kuczyk MA and Serth J: Prognostic and diagnostic relevance of hypermethylated in cancer 1 (HIC1) $\mathrm{CpG}$ island methylation in renal cell carcinoma. Int $\mathrm{J}$ Oncol 40 1650-1658, 2012.

4. Morris MR, Ricketts C, Gentle D, Abdulrahman M, Clarke N, Brown M, Kishida T, Yao M, Latif F and Maher ER: Identification of candidate tumour suppressor genes frequently methylated in renal cell carcinoma. Oncogene 29: 2104-2117, 2010.

5. van Vlodrop IJ, Baldewijns MM, Smits KM, Schouten LJ, van Neste L, van Criekinge W, van Poppel $H$, Lerut E, Schuebel KE, Ahuja N, et al: Prognostic significance of Gremlin1 (GREM1) promoter CpG island hypermethylation in clear cell renal cell carcinoma. Am J Pathol 176: 575-584, 2010.

6. Kawai Y, Sakano S, Suehiro Y, Okada T, Korenaga Y, Hara T, Naito K, Matsuyama $\mathrm{H}$ and Hinoda $\mathrm{Y}$ : Methylation level of the RASSF1A promoter is an independent prognostic factor for clear-cell renal cell carcinoma. Ann Oncol 21: 1612-1617, 2010.

7. Lasseigne BN, Burwell TC, Patil MA, Absher DM, Brooks JD and Myers RM: DNA methylation profiling reveals novel diagnostic biomarkers in renal cell carcinoma. BMC Med 12: 235 , 2014.

8. Arai E, Ushijima S, Tsuda H, Fujimoto H, Hosoda F, Shibata T, Kondo T, Imoto I, Inazawa J, Hirohashi $\mathrm{S}$ and Kanai Y: Genetic clustering of clear cell renal cell carcinoma based on array-comparative genomic hybridization: Its association with DNA methylation alteration and patient outcome. Clin Cancer Res 14: 5531-5539, 2008.
9. Ricketts CJ, Morris MR, Gentle D, Shuib S, Brown M, Clarke N, Wei W, Nathan P, Latif F and Maher ER: Methylation profiling and evaluation of demethylating therapy in renal cell carcinoma. Clin Epigenetics 5: 16, 2013.

10. Slater AA, Alokail M, Gentle D, Yao M, Kovacs G, Maher ER and Latif F: DNA methylation profiling distinguishes histological subtypes of renal cell carcinoma. Epigenetics 8: 252-267, 2013.

11. Wei JH,Haddad A,Wu KJ,ZhaoHW, Kapur P,Zhang ZL,ZhaoLY Chen ZH, Zhou YY, Zhou JC, et al: A CpG-methylation-based assay to predict survival in clear cell renal cell carcinoma. Nat Commun 6: 8699, 2015.

12. Arai E, Kanai Y, Ushijima S, Fujimoto H, Mukai K and Hirohashi S: Regional DNA hypermethylation and DNA methyltransferase (DNMT) 1 protein overexpression in both renal tumors and corresponding nontumorous renal tissues. Int J Cancer 119: 288-296, 2006.

13. Ellinger J, Kahl P, Mertens C, Rogenhofer S, Hauser S, Hartmann W, Bastian PJ, Büttner R, Müller SC and von Ruecker A: Prognostic relevance of global histone H3 lysine 4 (H3K4) methylation in renal cell carcinoma. Int J Cancer 127: 2360-2366, 2010.

14. Greene FL: The American Joint Committee on Cancer: Updating the strategies in cancer staging. Bull Am Coll Surg 87: 13-15, 2002.

15. Lopez-Beltran A, Scarpelli M, Montironi R and Kirkali Z: 2004 WHO classification of the renal tumors of the adults. Eur Urol 49: 798-805, 2006.

16. Fuhrman SA, Lasky LC and Limas C: Prognostic significance of morphologic parameters in renal cell carcinoma. Am J Surg Pathol 6: 655-663, 1982

17. Baylin SB and Jones PA: A decade of exploring the cancer epigenome-biological and translational implications. Nat Rev Cancer 11: 726-734, 2011.

18. Ushijima T: Detection and interpretation of altered methylation patterns in cancer cells. Nat Rev Cancer 5: 223-231, 2005.

19. Krishna SS, Majumdar I and Grishin NV: Structural classification of zinc fingers: Survey and summary. Nucleic Acids Res 31: 532-550, 2003.

20. Jen $\mathrm{J}$ and Wang $\mathrm{YC}$ : Zinc finger proteins in cancer progression. J Biomed Sci 23: 53, 2016.

21. Cheng Y, Liang P, Geng H, Wang Z, Li L, Cheng SH, Ying J, Su X, Ng KM, Ng MH, et al: A novel 19q13 nucleolar zinc finger protein suppresses tumor cell growth through inhibiting ribosome biogenesis and inducing apoptosis but is frequently silenced in multiple carcinomas. Mol Cancer Res 10: 925-936, 2012.

22. Deng J, Liang H, Ying G, Dong Q, Zhang R, Yu J, Fan D and Hao $X$ : Poor survival is associated with the methylated degree of zinc-finger protein 545 (ZNF545) DNA promoter in gastric cancer. Oncotarget 6: 4482-4495, 2015.

23. Jen J, Lin LL, Chen HT, Liao SY, Lo FY, Tang YA, Su WC, Salgia R, Hsu CL, Huang HC, et al: Oncoprotein ZNF322A transcriptionally deregulates alpha-adducin, cyclin D1 and p53 to promote tumor growth and metastasis in lung cancer. Oncogene 35: 2357-2369, 2016.

24. Yang L, Hamilton SR, Sood A, Kuwai T, Ellis L, Sanguino A, Lopez-Berestein G and Boyd DD: The previously undescribed ZKSCAN3 (ZNF306) is a novel 'driver' of colorectal cancer progression. Cancer Res 68: 4321-4330, 2008

25. Bar-Shavit R, Maoz M, Kancharla A, Nag JK, Agranovich D, Grisaru-Granovsky S and Uziely B: G protein-coupled receptors in cancer. Int J Mol Sci 17: E1320, 2016.

26. Lappano R and Maggiolini M: G protein-coupled receptors: Novel targets for drug discovery in cancer. Nat Rev Drug Discov 10: 47-60, 2011. 